\title{
Analysis of Resonant Behavior in Planar Line Arrays of Rings by the Eigencurrent Approach
}

\author{
Dave J. Bekers ${ }^{1}$, Stef J.L. van Eijndhoven ${ }^{2}$, Alphons A.F. van de Ven ${ }^{3}$, Peter-Paul Borsboom ${ }^{4}$, Anton G. Tijhuis ${ }^{5}$ \\ ${ }^{1}$ TNO Defence, security, and safety, Observation Systems, Oude Waalsdorperweg 63, \\ PO Box 96864, 2509 JG The Hague, the Netherlands, (+31)(0)70 3740247, dave.bekers@tno.nl \\ ${ }^{2}$ Technische Universiteit Eindhoven, Department of Mathematics and Computer Science, Den Dolech 2, \\ 5600 MB Eindhoven, the Netherlands, (+31)(0)40 2472808, s.j.l.v.eijndhoven@tue.nl \\ ${ }^{3}$ Technische Universiteit Eindhoven, Department of Mathematics and Computer Science, Den Dolech 2, \\ 5600 MB Eindhoven, the Netherlands, (+31)(0)40 2472803, a.a.f.v.d.ven@tue.nl \\ ${ }^{4}$ SARA Computing and Networking Services, Kruislaan 415, Postbox 94613, 1090 GP, \\ Amsterdam, the Netherlands, peter-paul@sara.nl \\ ${ }^{5}$ Technische Universiteit Eindhoven, Department of Electrical Engineering, Den Dolech 2, \\ 5600 MB Eindhoven, the Netherlands, (+31)(0)40 2473800, a.g.tijhuis@tue.nl
}

\begin{abstract}
Resonant behavior in a finite array that appears as (modulated) impedance or current-amplitude oscillations may limit its bandwidth substantially. Therefore, simulations should predict such behavior. Recently, a new approach has been developed, called the eigencurrent approach, which can predict resonant behavior in finite arrays. Analysis of line arrays of $H$-plane oriented microstrip rings by the eigencurrent approach reveals that resonant behavior is caused by excitation of one of the eigencurrents. The characteristic impedance of this eigencurrent becomes small in comparison to the characteristic impedances of the other eigencurrents that can exist on the array geometry.
\end{abstract}

\section{INTRODUCTION}

The design and development of antenna arrays is complex and costly. To reduce design costs and design risks, and to improve the performance of the arrays, simulations are used. At present, brute-force numerical approaches applied to a large array are still far too computationally expensive. Therefore, an array is often considered as an infinite periodic structure, where symmetry is used to restrict the analysis to a single antenna element of the array. This approach, however, cannot completely describe the characteristic electromagnetic behavior of antenna arrays. In particular, it cannot describe resonant behavior that limits their bandwidth severely. Such behavior appears as (modulated) impedance or currentamplitude oscillations as described in [4], [5], and [6]. These oscillations or variations may decrease the performance of arrays considerably. To explain this, we mention that in various types of arrays, certain groups of elements are matched with the same impedance to the feeding network. An example is a rectangular array, which is excited per row of elements. In that case, the rows constitute the different groups. If the elements of a row are matched with the same impedance to the feeding network, while large variations in element impedances occur, the result is huge differences in energy reflection into the feeding network. This energy is partly lost in the network. Moreover, via the network, reflected energy of an element is partly transferred to other elements, which in turn radiate or reflect this energy. Consequently, the total radiated power decreases and the far field is distorted. Therefore, it is important to predict resonant behavior accurately. In [2], we proposed an approach that describes the characteristic behavior of finite arrays accurately and leads to rapidly executable simulations. The approach, which we called the eigencurrent approach, can predict the mentioned resonant behavior.

In this paper, we first introduce briefly the main ideas of the eigencurrent approach. For a detailed account, we refer to [2]. Next, we demonstrate resonant behavior in planar uniform line arrays of $\mathrm{H}$-plane oriented microstrip rings and we analyze this behavior by the eigencurrent approach. In particular, we show that resonant behavior is caused by the excitation of specific eigencurrents or eigenfunctions of the array. In a forthcoming paper [3], we go into detail with respect to the (physical) explanation of resonant behavior in finite arrays. We test our findings for line arrays of rings and line arrays of strips, both in free space and in a half space, where we compare with results in the literature. Finally, we show that resonant behavior persists when the geometry of a uniform array is slightly perturbed, as is the case in practice.

\section{The EIGENCURRENT APPROACH}

In the eigencurrent approach, the behavior of a finite array is described by its eigenvibrations or eigencurrents. These eigencurrents are the eigenfunctions of the impedance operator that relates the currents on the elements to their excitation fields, which are induced by a plane wave or local sources, for example. From a physical point of view, the eigencurrents are standing waves of the array. The corresponding eigenvalues represent the characteristic impedances of the eigencurrents. The larger the characteristic impedance of an eigencurrent, the less this eigencurrent contributes to the current on the elements for a given excitation field. The concept of eigencurrent appears extremely suitable for the design of arrays, because the design characteristics and the excitation of specific eigencurrents are one-to-one related. The spectral analysis of the impedance operator in [2] reveal that eigencurrents and corresponding eigenvalues are one-to-one related to sum patterns, to difference patterns, to grating lobes, to modulated impedance oscillations, to impedance variations attributed to surface waves, and to many other properties of the array.

Starting point of the approach is the determination of the spectrum of a single element. Eigenvalues and eigencurrents 
are computed from a 'normalized' moment matrix related to chosen expansion functions for the current on the element. If the eigencurrents of the element are known, this first step is unnecessary. An example of such an element is the ring, as considered in the next section, of which the eigencurrents are $1, \cos n \varphi, \sin n \varphi(n=1,2, \ldots)$, where $\varphi$ is the angle that describes the circumference of the ring. Subsequently, an inner product is determined for which the single-element eigencurrents are orthonormal. The corresponding moment matrix in terms of these eigencurrents is a diagonal matrix with respect to the new inner product. In the second step, a reduced moment matrix for the array is computed with respect to the composition of the new element inner products, with as expansion functions the eigencurrents per element. Only a limited number of single-element eigencurrents that contribute to mutual coupling in the array are taken into account, see [2] for details. The results of the second step are the array eigencurrents, described as concatenations of linear combinations of coupling single-element eigencurrents. The array eigencurrents are divided into groups, where each group corresponds to one dominant single-element eigencurrent. This eigencurrent describes the dominant behavior of the array eigencurrents in a group. The eigenvalues in a group evolve from the eigenvalue of the corresponding dominant singleelement eigencurrent.

By the eigencurrent approach, the current $\boldsymbol{J}$ on the elements of a finite array is found as

$$
\boldsymbol{J}=\sum_{n=1}^{N} \sum_{q=1}^{Q} \frac{1}{\nu_{n q}}\left\langle\boldsymbol{u}_{n q}, \boldsymbol{E}^{\mathrm{ex}}\right\rangle \boldsymbol{u}_{n q} .
$$

Here, $\left\{\nu_{n q}\right\}_{q=1}^{Q}$ and $\left\{\boldsymbol{u}_{n q}\right\}_{q=1}^{Q}(n=1, \ldots, N)$ are the groups of eigenvalues and corresponding groups of eigencurrents, $\boldsymbol{E}^{\mathrm{ex}}$ is the tangential excitation field on the elements of the array, and $Q$ is the number of elements. Moreover, $N$ is the number of single-element eigencurrents, which depends on the number of expansion functions used to construct the moment matrix at the start of the approach. From the expression (1), it is clear that a group of eigenvalues $\left\{\nu_{n q}\right\}_{q=1}^{Q}$ corresponding to a larger single-element eigenvalue in general contributes less to the current than a group of eigenvalues corresponding to a smaller single-element eigenvalue. The ring elements we consider in this paper are typically designed to excite a single eigencurrent. Consequently, the current on the rings of an array is predominantly described by the first group of eigenvalues ( $n=1)$, where we index the groups according to increasing single-element eigenvalues.

\section{RESONANT BEHAVIOR IN ARRAYS}

As an illustration, we consider a uniform line array of 40 microstrip rings in free space with a spacing equal to three times the ring radius, see Figure 1 . The rings are excited by voltage gaps of $1 \mathrm{~V}$ positioned on the array, by which the rings are $H$-plane oriented. All rings are equally phased. The width $b$ of the rings is much smaller than the wavelength, while the spacing $d$ and the radius $a$ are of the same order as the wavelength. The surfaces of the rings are modeled as perfectly conducting and infinitely thin. Moreover, the currents on the rings are averaged with respect to their widths, see [1] or [2, Ch. 2]. Consequently, these currents are described by integrodifferential equations with logarithmically singular kernels.

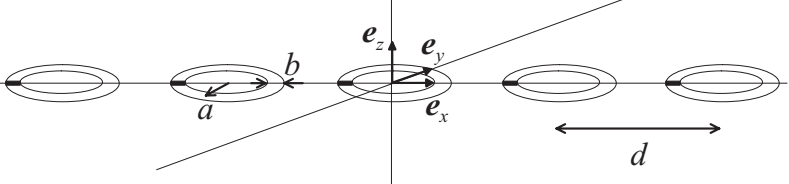

Fig. 1. A line array of rings excited by voltage gaps indicated by black bars.
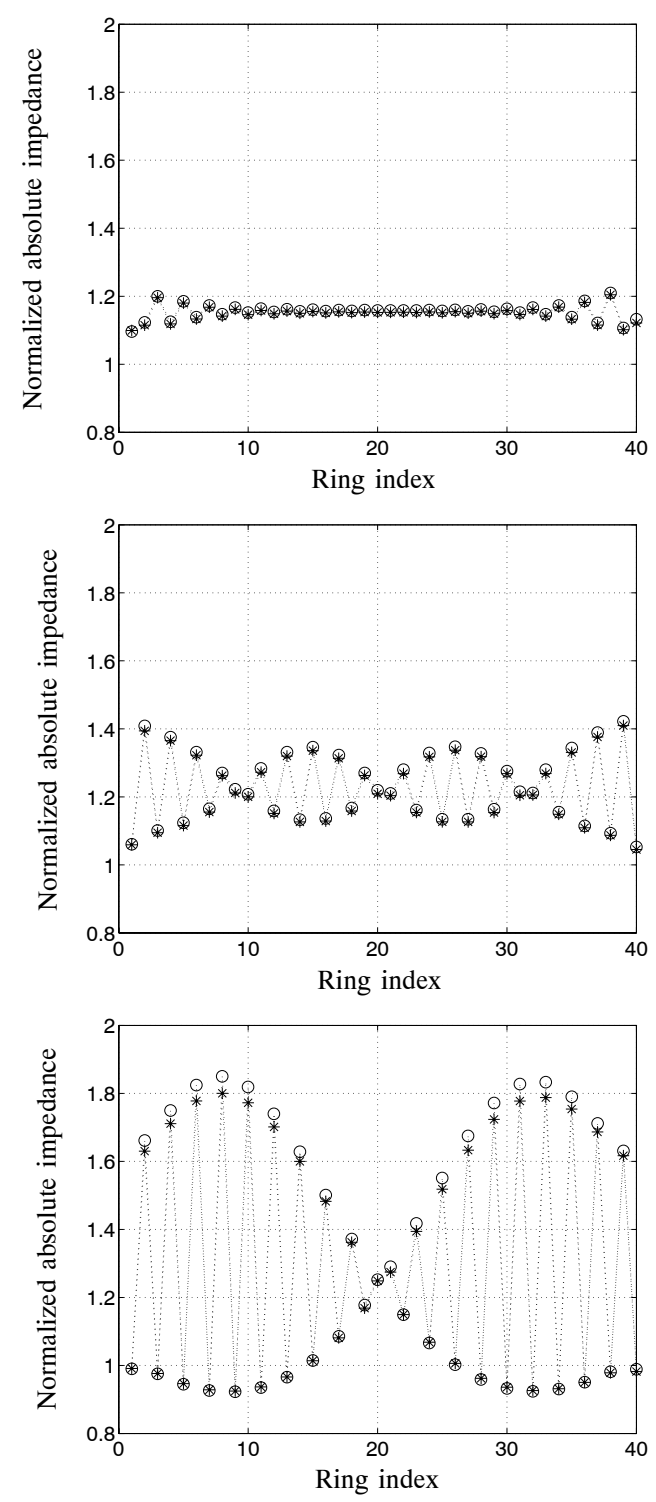

Fig. 2. Normalized absolute ring impedances for a line array of 40 (H-plane oriented) rings in free space excited by voltage gaps of $1 \mathrm{~V}$ for a scan at $0^{\circ}$. Upper figure: frequency with $k a=1.047$. Middle figure: $k a=0.943$. Lower figure: $k a=0.971$. Impedances computed by the moment method $(*)$ and by the eigencurrent approach (०). Normalization: for each frequency, the corresponding absolute impedance of a single ring. Parameter values: $d / a=3$ $(d=\lambda / 2$ at $k a=1.047), b / a=3 / 100$.

In Figure 2, we depict the normalized absolute impedances of the rings for the frequencies with $k a=1.047, k a=0.943$, and $k a=0.971$, both computed by the moment method and by the eigencurrent approach. For $k a=1.047$, the impedance pattern is almost uniform, while for the other two frequencies, the impedance pattern exhibits relatively large modulated oscillations. Moreover, for $k a=0.971$, the period of the modulations is shorter than for $k a=0.943$. Notice that 
the results obtained by the eigencurrent approach and by the moment method match very well.

The same kind of modulated oscillations are discussed in [4] for arrays of collinear, or $E$-plane oriented, wires with spacing $\lambda / 2$ in a half space with $h=\lambda / 4$, where $h$ is the height above the ground plate. The dipole length is chosen such that the elements exhibit a 'resonant broadside embedded impedance', i.e., the reactance of the elements equals on average zero. The modulated oscillations are not observed for arrays of parallel, or $H$-plane oriented, wires, neither for arrays in free space. In contrast, the rings in Figure 2 are positioned in free space and are $H$-plane oriented, since the voltage gaps are all positioned on the array axis. Moreover, the spacing is not equal to $\lambda / 2$; for $k a=0.971$ and $k a=0.943$, the spacing is $0.464 \lambda$ and $0.450 \lambda$, respectively. Since the ring array is positioned in free space, the modulated oscillations cannot be explained by a traveling wave between the array and a conducting ground plane, as suggested in [4] and [5].

The large modulated oscillations are explained by the eigencurrent approach. Figure 3 shows the normalized absolute expansion coefficients in the finite expansion (1) of the current for the above mentioned three values of $k a$. Only the coefficients of the eigencurrents of the first group are shown, i.e., $\left\{\left\langle\boldsymbol{u}_{1 q}, \boldsymbol{E}^{\mathrm{ex}}\right\rangle / \nu_{1 q}\right\}_{q=1}^{40}$. We note that these eigencurrents are

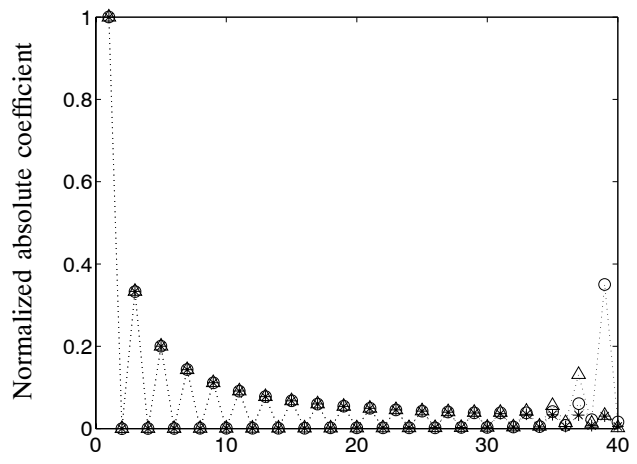

Eigencurrent index

Fig. 3. Normalized absolute coefficients in the finite expansion (1) of the current on the line array of 40 rings in Figure 2 for the frequencies with $k a=0.943(\triangle), k a=0.971(\circ)$, and $k a=1.047(*)$. Only the coefficients of the eigencurrents of the first group are shown. Normalization: maximum absolute coefficient.

indexed along the curve that their corresponding eigenvalues describe in the complex plane. This is illustrated further on in Figure 5. For $k a=1.047$, the coefficients of eigencurrents with even indices are zero, while the coefficients of odd eigencurrents form a monotonically decreasing sequence. The coefficients of eigencurrents with even indices are zero, because these eigencurrents exhibit an odd phase distribution along the array. More precisely, the coefficients of their dominant singlering eigencurrent exhibit an odd phase distribution along the array. For $k a=0.971$ and $k a=0.943$, the coefficients show the same behavior as the coefficients for $k a=1.047$, but the 39th and 37th (array) eigencurrents, respectively, have a higher coefficient. Figure 4 shows the absolute values and the phases of the coefficients of the dominant single-ring eigencurrent in the 39th array eigencurrent. We note that the dominant single-ring eigencurrent is in this case $\cos \varphi$, since the ring circumference is about one wavelength. The absolute values show an absolute sine-like pattern, while the phase distribution shows that each ring has opposite phase with respect to its
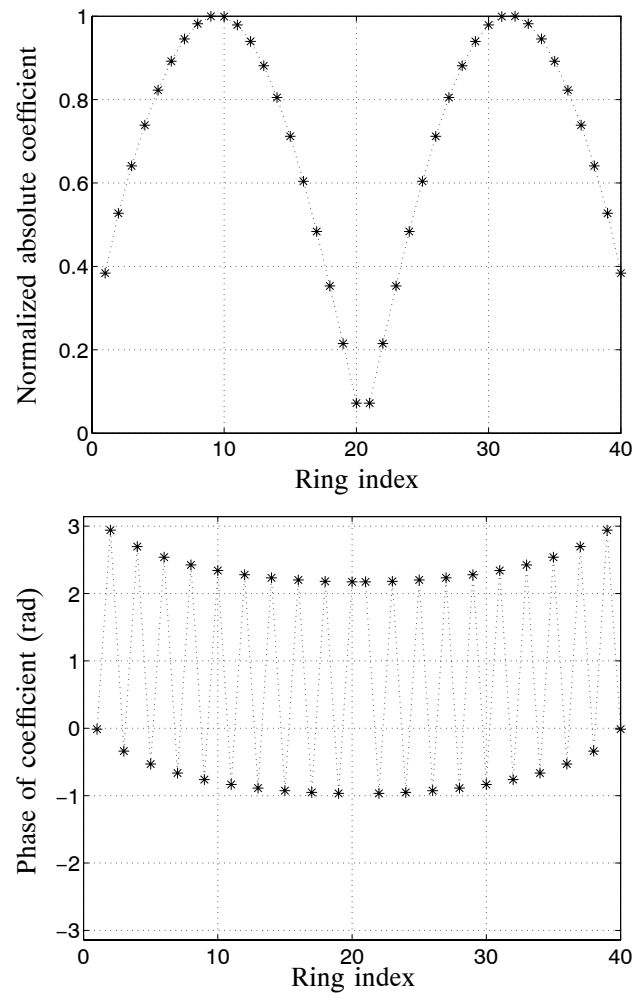

Fig. 4. Normalized absolute values (upper figure) and phases (lower figure) of the coefficients of the dominant single-ring eigencurrents in the 39th eigencurrent of the first group for the array in Figure 2. Normalization: maximum absolute coefficient.

neighbors, except for the 20th and 21st ring. Consequently, the coefficients exhibit an alternating pattern modulated by a sine of one period. This pattern can be observed clearly in the impedance pattern of Figure 2 (lower figure). Similarly, the coefficients of the dominant single-ring eigencurrents 37 th array eigencurrent show an alternating pattern modulated by a sine of two periods, which can be observed clearly in the impedance pattern of Figure 2 (middle figure).

One might think that the excitation of the 37th and 39th eigencurrent is due to differences between the eigencurrents at $k a=0.943, k a=0.971$, and $k a=1.047$. However, in [2], we showed that the coefficients of the dominant single-element eigencurrent in the array eigencurrents depend negligibly on the element shape and the frequency. Moreover, the singleelement eigencurrents of a ring do not depend on the ring geometry and the frequency. Hence, the excitation of the 37th and the 39th eigencurrent is not due to changes of the eigencurrents. Moreover, when the inner products $\left\langle\boldsymbol{u}_{1 q}, \boldsymbol{E}^{\mathrm{ex}}\right\rangle$ are plotted, the little peaks in Figure 3 disappear. Hence, these peaks are caused by a change of the eigenvalues.

Figure 5 shows the behavior of the eigenvalues in the complex plane and the corresponding behavior of their absolute values for varying frequency. The minimum absolute eigenvalues for $k a=0.943$ and $k a=0.971$ are the 37 th and 39th eigenvalue, which correspond to the curves in Figure 3 with the small peaks at the 37 th and 39th eigencurrents, respectively. In comparison to the other eigenvalues, these eigenvalues are close to zero. In other words, the 37th and 39th eigencurrent exhibit a small characteristic impedance in comparison to the characteristic impedances of the other eigencurrents. Therefore, they are excited despite the fact that 
